\title{
3D Scan to Product Design: Methods, Techniques, and Cases
}

\author{
Wonsup LEE*1 ${ }^{1}$, Baekhee LEE ${ }^{2}$, Sungho KIM², Hayoung JUNG ${ }^{2}$, \\ Eunjin JEON ${ }^{2}$, Teukgyu $\mathrm{CHOI}^{3}$, Heecheon $\mathrm{YOU}^{2}$ \\ ${ }^{1}$ Faculty of Industrial Design Engineering, Delft University of Technology, Delft, The Netherlands; \\ ${ }^{2}$ Department of Industrial and Management Engineering, Pohang University of Science and \\ Technology, Pohang, Korea; ${ }^{3}$ Humanopia Inc., Pohang, Korea
}

DOI: $10.15221 / 15.168 \quad$ http://dx.doi.org/10.15221/15.168

\begin{abstract}
3D scanning technology has derived great opportunities for ergonomic product designs. This paper is aimed to introduce various research cases and methods based on 3D scanning have conducted by an ergonomics laboratory in South Korea. Sizing systems and representative 3D models developed based on anthropometric measurements and 3D scan images with technical know-how were applied to the design of various products. Head, face, ear, upper limb, and waist parts, and full body in seated posture were anthropometrically analyzed for the design of headwear (e.g., helmet, goggle, and headphone), oxygen mask, earphone, arm-wear (e.g., watch, armband), hip protector, and vehicle seat, respectively. Customized software for the efficient analyses such as measurement of anthropometric dimensions, analysis of sizing systems, extraction of representative models, and virtual fit evaluation between products and the body were developed and applied in the product design process with massive 3D scan images. Representative models (e.g., torso and head) were printed in $3 \mathrm{D}$ for effective usage to the design and evaluation of related products. Advanced methods and techniques such as finite element modeling, morphing, and skin deformation have been applied to 3D scanned images for an advanced design of product shapes in further researches.
\end{abstract}

Keywords: 3D scanning, anthropometric analysis, ergonomic product design

\section{Introduction}

3D scanning technology has derived great opportunities for ergonomic product designs. Based on the 3D scan, not only simple dimensions (e.g., length, circumference) also complex dimensions such as section curve, surface shape, area, and volume which can provide useful information for the product design can be measured $[1,2,3,4,5]$. Furthermore, 3D scanned images have been applied to development of sizes of a product (e.g., small, medium, and large), generation of representative human models, and 3D printing of body parts (e.g., headform, torso) for the efficient progress in the product design process $[6,7,8,9]$. A sizing system generated based on massive $3 \mathrm{D}$ human scan images supports summarized information of target population (e.g., number of sizes of a product, accommodation percentage). A digital or 3D printed representative model provides the anthropometric characteristics (e.g., size, shape in 3D) of the target population of a product. Recently, advanced computer techniques such as automatic measurement of anthropometric dimensions, skin deformation, posture change, virtual fitting, prediction of contact pressure, or estimation of muscle force have been applied to the 3D scan data $[4,10,11,12,13,14]$.

Ergonomic Design Technology (EDT) laboratory of Pohang University of Science and Technology (POSTECH) [15] in South Korea has many year successful experiences on the ergonomic product design and development cooperated with various organizations. Anthropometric information, digital human models, and 3D body scan images have been applied to the design of various products such as Korean utility helicopter's cockpit [16], driver's seat of vehicle [17], layout of control room of the radioactive waste management facility [18], size of radar console [19], vacuum cleaner's handle and stick, flight suit and oxygen mask for Korean Air Force pilots [3], hip protector for elderly women [20], head and face wearable products [8], ear-related products [21], and dust-proof mask. 3D scan images and $3 \mathrm{D}$ anthropometric information have been utilized to the product designs through whole or part of a five-step process: (1) establishment of 3D human scan database, (2) analysis of anthropometric measurements, (3) development of sizing system, (4) development of representative models, and (5) design of size and shape of product. The aim of this paper is to introduce methods, techniques, and successful research cases related to the ergonomic product design based on 3D human body scanning.

\footnotetext{
*w.lee@tudelft.nl; +31 (0) 152782879
} 


\section{Product design based on 3D scanned human body}

\subsection{Establishment of 3D human scan database}

3D human body images were prepared by 3D scanning (e.g., face, ear, full body in seating posture, inside of hand in grasp posture, deformed body part) or by applying existing 3D scan databases established by Civilian American and European Surface Anthropometry Research (CAESAR) [22] or Size Korea survey [23] (see figure 1). First, 336 Korean Air Force pilots' face (male: 278, female: 58, age: $20 \mathrm{~s} \sim 40 \mathrm{~s}$ ) were 3D scanned and measured for the design of mask-related products (e.g., pilot oxygen mask) [3,4]. Second, 200 Koreans (male: 100, female: 100) and 96 Caucasians (male: 46, female: 50 ) aged 20 to 59 were 3D scanned for the design of ear-related products. After the overall ear shape was directly scanned in 3D and the concha with inside of ear canal parts was cast by applying the ear casting materials then scanned in 3D, the two scanned parts (overall ear and concha) were merged as a complete ear. The sample sizes in the face and ear measurement studies were decided based on an equation suggested by ISO [24]. Third, 3D heads extracted from 2,299 North American CAESAR (male: 1,086, female: 1,213, age: $18 \sim 70$ s) were established as a 3D head database for the purpose of head-related product (e.g., helmet, mask, goggle, and headphone) designs [8]. Because the CAESAR 3D images have large misscanned parts on their head and unnaturally volumized hair, all the 3D heads were manually edited for the useful application to the product design. Fourth, 3D torso images of 271 Korean female elders (age: $60 \sim 75$ ) scanned by 2004 Size Korea survey were edited and measured for the design of hip protector which can prevent a hip fracture from the falling accident. Lastly, for a detailed analysis on specific part of a product, a corresponding body part in certain posture was scanned. For example as shown in figure 2, seated posture without deformation on the buttock was scanned for the application to a vehicle seat design. Inside of hand with a grasping posture was scanned for a handgrip design. And the face deformed by a flat glass was scanned to find a characteristic of facial skin deformation.

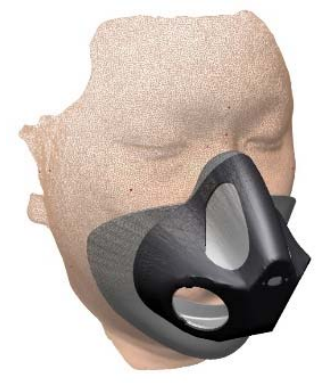

(a) 3D face image of Korean Air Force pilot $(N=336)$

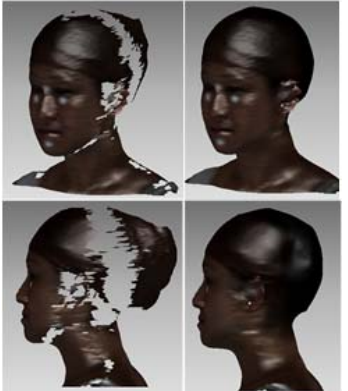

(b) the North American CAESAR 3D head before and after editing $(N=2,299)$

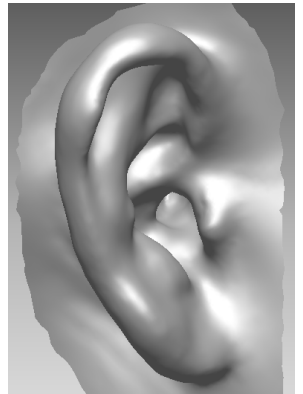

(c) 3D ear image including ear canal $(N=326)$
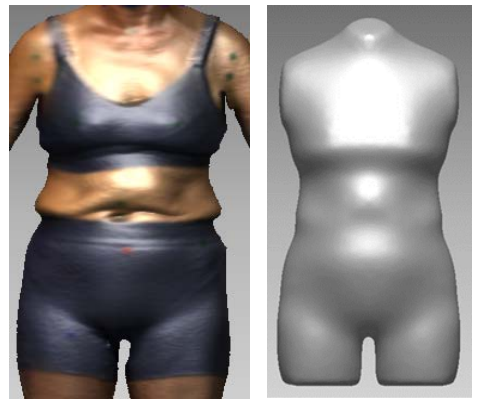

(d) 3D torso image of Korean female elder $(N=271)$

Fig. 1. 3D scanned body parts (illustrated)

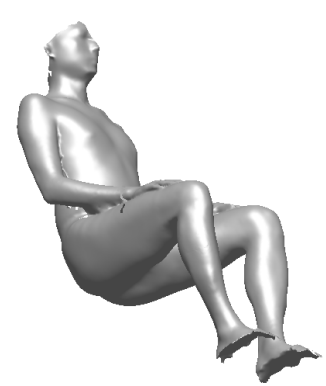

(a) seated posture without deformation on the buttock

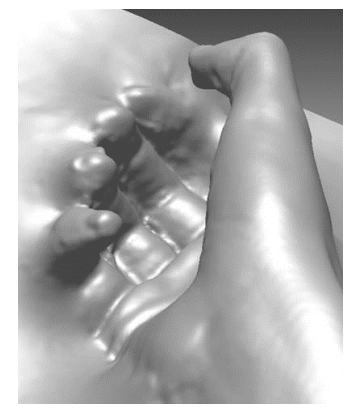

(b) inside of hand in grasp posture

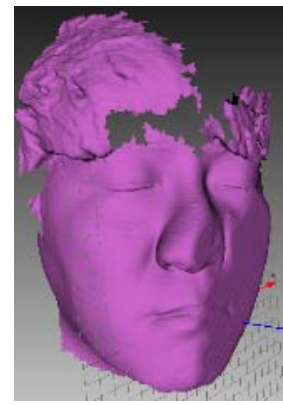

(c) deformed face

Fig. 2. Specialized $3 D$ scanning for detailed analyses of the body part having posture or deformation (illustrated)

\subsection{Analysis of anthropometric measurements}

Anthropometric measurement dimensions related to the product design were selected through a review of literature and the recommendation of a panel of experts. For example, out of 122 facial dimensions which were identified by eighteen previous studies, 22 facial dimensions and 14 landmarks 
related to the measurement were selected for the design of pilot oxygen mask [3] and 28 head and face dimensions and 25 landmarks were chosen for the design of head-related products [8]. For the ear measurement, 17 ear dimensions and 16 corresponding landmarks were selected by referring to 21 previous studies [21]. For hip protector design, 20 related body dimensions were measured based on 3D images of Korean female elders. After anthropometric dimensions were selected, anthropometric landmarks were manually identified on the 3D scan images by referring to landmarking protocols proposed by the previous anthropometric researches. Some of manually identified landmarks are used to find virtual landmarks such as center of ear canal entrance, which cannot be located on the skin. The dimensions were automatically measured using a measurement program developed with Matlab (MathWorks, Inc., Natick, MA, USA) in our studies. Once the measurement was made, the integrity of measurements using $3 \mathrm{D}$ scan was assured by an outlier checking process. Then the measurements were statistically analyzed and compared between sample groups or compared to the existing measurements by applying statistical techniques such as $t$ - and $F$-test, or by $z$-test with pooled mean and standard deviation.

\subsection{Development of sizing system}

Anthropometric measurements were applied to the generation of an optimal sizing system by applying various statistical techniques (e.g., factor analysis, principal component analysis, and regression analysis) and a distributed method (e.g., grid method, optimization method, and clustering method) [25]. A five-step protocol (S1. target population selection, S2. target anthropometric variable selection, S3. extraction of key dimensions, S4. determination of a distributed method, S5. Generation of a sizing system) for the exploration of an optimal sizing system was applied to the design of flight suit [26] and pilot oxygen mask [4] for Korean Air Force pilots, hip protector [20], earphone [21], gloves [27], dust-proof mask, and so on. Once candidates of a sizing system are generated through the protocol based on statistic techniques, the candidates are compared in terms of accommodation percentage, number of sizes, and coverage ratio in non-key-dimensions [25]. Then a selected sizing system is optimized to improve accommodation percentage and ease-of-use by adjusting range of sizes through the recommendation of a panel of experts (see figure 3 ).

\begin{tabular}{|c|c|c|c|c|c|}
\hline $\begin{array}{l}\text { Stature } \\
\text { Chest } \\
\text { circumference }\end{array}$ & $\begin{array}{l}\text { Small } \\
<168\end{array}$ & $\underset{<173}{\text { Medium }}$ & $\begin{array}{l}\text { Large } \\
<178\end{array}$ & $\begin{array}{c}\text { Special } \\
<183\end{array}$ & $\begin{array}{c}\text { Special } \\
\text { large } \\
<190\end{array}$ \\
\hline $\begin{array}{c}85 \\
(82.5 \sim 87.4)\end{array}$ & $0.9 \%$ & $3.2 \%$ & $2.1 \%$ & & \\
\hline $\begin{array}{c}90 \\
(87.5 \sim 92.4)\end{array}$ & $1.6 \%$ & $7.2 \%$ & $8.1 \%$ & $2.9 \%$ & \\
\hline $\begin{array}{c}95 \\
(92.5 \sim 97.4)\end{array}$ & $1.9 \%$ & $10.1 \%$ & $12.2 \%$ & $5.5 \%$ & $1.1 \%$ \\
\hline $\begin{array}{c}100 \\
(97.5 \sim 102.4)\end{array}$ & $0.9 \%$ & $5.3 \%$ & $10.8 \%$ & $6.8 \%$ & $2.1 \%$ \\
\hline $\begin{array}{c}105 \\
(102.5 \sim 107.4)\end{array}$ & & $2.2 \%$ & $4.2 \%$ & $3.5 \%$ & $1.2 \%$ \\
\hline $\begin{array}{c}110 \\
(107.5 \sim 112.4)\end{array}$ & & & $1.5 \%$ & $1.2 \%$ & \\
\hline
\end{tabular}

(a) sizing system of flight suit for Korean Air Force pilots and accommodation percentages of each size (23 sizes)

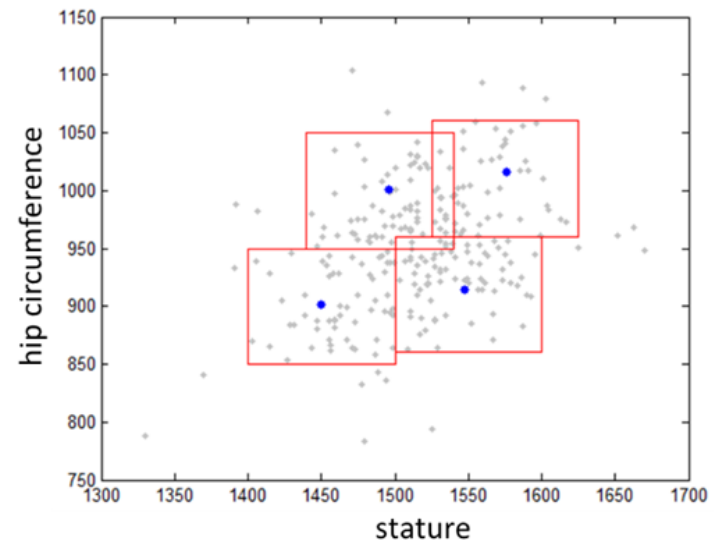

(c) sizing system of hip protector for Korean elders (4 sizes; blue dot: representative model)

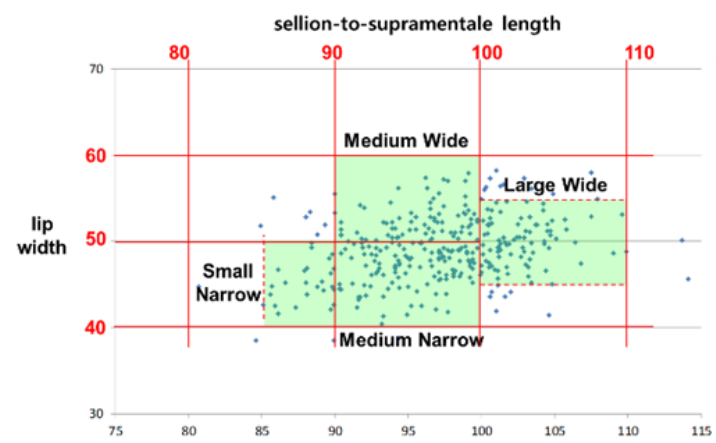

(b) sizing system of Air Force pilot oxygen mask (4 sizes)

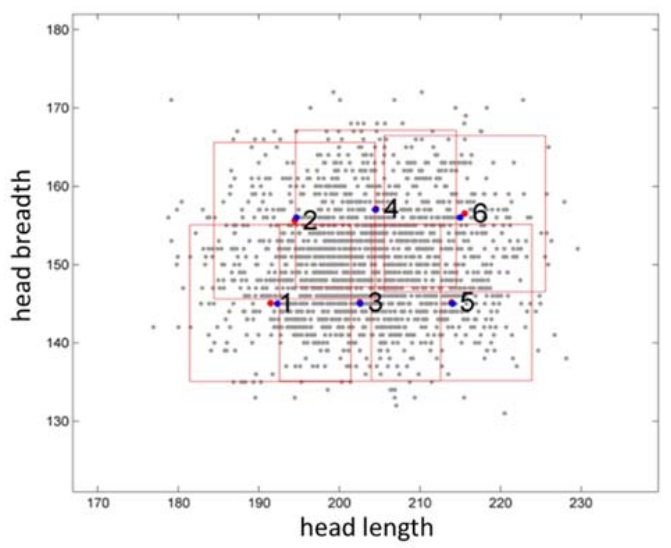

(d) sizing system of headwear design for Caucasians ( 6 sizes; blue dot: representative model)

Fig. 3. Examples of sizing system 
An anthropometric sizing analysis system (see figure 4) was developed for the efficient analysis of sizing systems [8]. For example of the sizing analysis for head-related product designs, a system developed based on the North American CAESAR database. The system can be used through 4-step process. First, target head-related product (e.g., helmet, mask, goggle, and headphone) is selected. Second, characteristics of target population are defined in terms of ethnic group, gender, and age group (e.g., 20s, 30s, 40s, 50s, and 60s). Third, one or two key anthropometric dimensions corresponding to design dimensions of the target product are selected. Finally, based on statistical analysis [25], an optimized sizing system is generated by adjusting of a number of sizes, range of coverage sizes, and accommodation percentage.

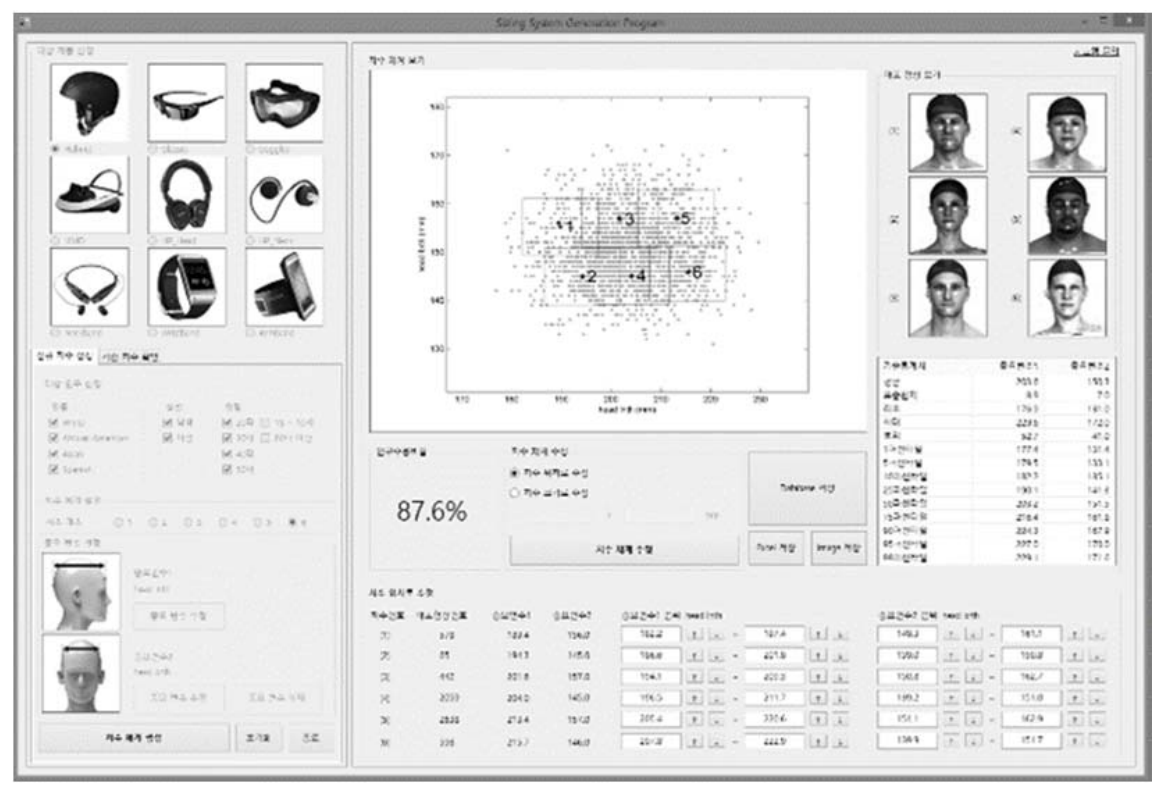

Fig. 4. An anthropometric sizing analysis system for head-related product designs (illustrated)

\subsection{Development of representative human models}

Representative models of human body parts (e.g., face, head, torso, ears) were generated and printed in $3 \mathrm{D}$ as shown in figure 5 for effective usage to the design and evaluation of related products. For example, based on the North American CEASAR database, 15 representative heads in terms of ethnic group and gender were identified and those heads were reproduced as headforms [8]. Average sizes of head and facial measurements in terms of each ethnic group (composite ethnic group, Caucasian, African American, Asian, and Hispanic) and gender (composite gender, male, and female) were calculated by applying the anthropometric sizing analysis system. Based on those measurements per group, 15 heads which have the most similar sizes from the average were found. To find the most representative heads from the dataset, a sum of weighted Euclidean distance calculated based on absolute differences between measurements of each head and the average values on all head dimensions with importance of each dimension was used [25]. Those heads were manually reproduced as digital headforms through RapidForm 2006 (INUS Technology, Inc., South Korea). All 15 heads were smoothed and mirrored; all measurements were adjusted to fit the average sizes; and 3D ears which have relevant size to the heads were attached to the average location of the ear on the head. Then, the headforms were 3D printed and polished for the use of product design and evaluation.
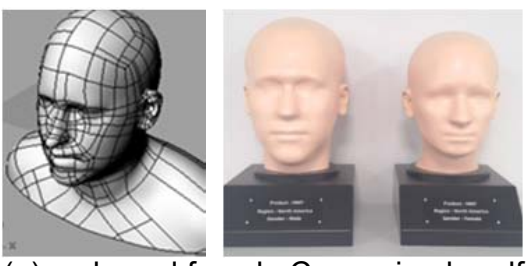

for glasses-type product designs

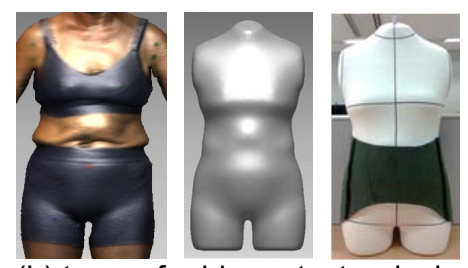

(b) torsos for hip protector design

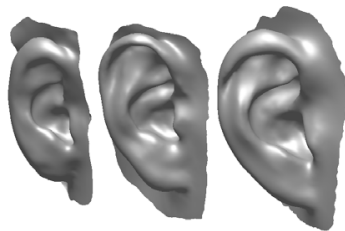

(c) ears for ear-related product designs

Fig. 5. Digital and/or 3D printed representative models (illustrated) 
For example of the hip protector design, four torsos of Korean elderly women were selected, edited, and printed in 3D, which are corresponded to its sizing system as shown in figure 3.c [20]. Because the not all dimensions of the representative model are same to the average value, the model can be slightly adjusted by applying a mesh deformation technique such as bounded biharmonic weights (BBW) deformation [12].

\subsection{Design of size and shape of product}

Three design approaches (e.g., design based on the representative models, design based on virtual fit technique, and design based on massive 3D shapes) have been utilized in our studies. The representative models were usually applied to the design of relatively flexible products such as clothing (e.g., hip protector) [20]. For example of the hip protector design, four representative models printed in $3 \mathrm{D}$ as a mannequin were used to a patternmaking through a draping procedure, then fit of the patterns was tested with those mannequins (see figure 6). Also the representative models were applied to create an initial shape of product, then the design was adjusted through an evaluation based on prototypes or a virtual fit testing. For example, four pilot oxygen masks were initially designed by referring to four corresponding representative faces of Korean Air Force pilots. Then the designs were adjusted to increase fit through an analysis based on virtual fit technique which virtually fits the oxygen mask CAD onto each $3 \mathrm{D}$ facial scan image $(N=336)$ and evaluates fit characteristic between the mask and the face [3]. The 3D full body scans in seated posture were applied to the design of a vehicle seat through the virtual fit analysis. Lastly, a size and shape of dust-proof mask was determined based on a massive data of 3D scan images. Contours intersecting the 3D face and the cross-sectional plane passing the sets of facial landmarks (e.g., bitragion-sellion, bitragion-pronasale, bitragion-lip, bitragion-menton) were superimposed, then size, shape, and range of design elements (e.g., length of mask, width of mask, curvature of the frontal part of mask, shape of the nose part of mask) were found based on the contours.

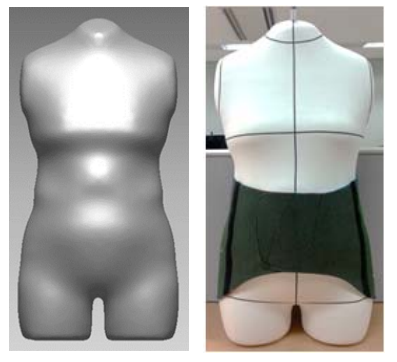

(a) design of hip protector based on the representative mannequin
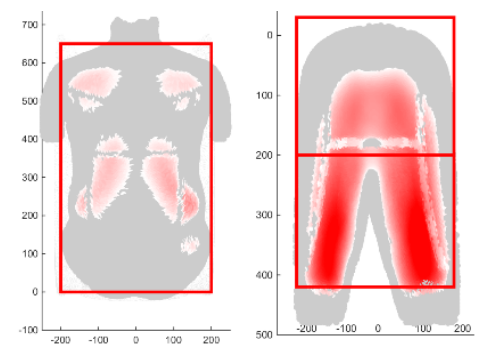

(d) design of vehicle seat based on virtual fit

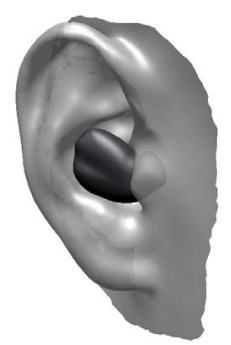

(b) design of initial size and shape of earphone based on the representative ear

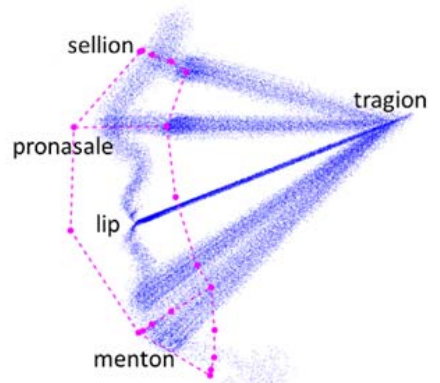

(e) design of dust -proof mask based on massive 3D shapes

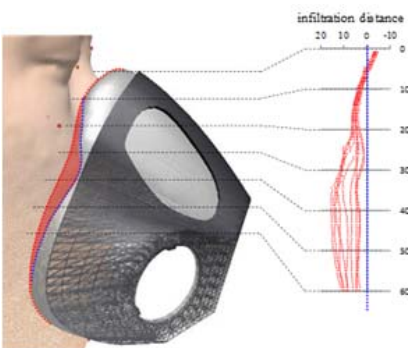

(c) design of pilot oxygen mask for Korean Air Force pilots based on virtual fit technique

Fig. 6. Design based on information generated based on $3 D$ body scan database (illustrated)

\section{Conclusion}

The five-step process for the ergonomic product design based on 3D human body scanning introduced in this paper is not far from the conventional product design procedure. However, because 3D human scan image which is digitalized and able to processed in computer software has more opportunity to be 
systematically manipulated, we have been tried to develop and utilize some technical methods on 3D scan images for the advanced application to the product design. Computerized systems and software which can provide useful anthropometric information (e.g., measurements, shape, sizing system, representative models) and systematic analyses (e.g., automatic measurement, shape analysis, fit analysis, sizing system development) were developed for the efficient progress in the product design process. Once the designs were proposed through our technical process, we have manufactured working prototypes for a design validation by applying a usability evaluation $[4,19,20,26]$. Also advanced methods and techniques such as finite element modeling, morphing, and skin deformation have been applied to 3D scanned images for an advanced design and evaluation of the product shapes in further researches.

\section{References}

[1] C. Chang et al., "Error control and calibration in three-dimensional anthropometric measurement of the hand by laser scanning with glass support", in Measurement, Vol.40, No.1, 2007, pp.21-27, DOI: 10.1016/j.measurement.2006.05.006.

[2] W. Lee et al., "Development of a 3D semi-automatic measurement protocol for hand anthropometric measurement", in Proc. of Human Factors and Ergonomics Society 54th Annual Meeting, San Francisco, CA, USA, 2008.

[3] W. Lee et al., "Analysis of the facial measurements of Korean Air Force pilots for oxygen mask design", in Ergonomics, Vol.56, No.9, 2013, pp.1451-1464, DOI: 10.1080/00140139.2013.816376.

[4] W. Lee et al., "Ergonomic design and evaluation of a pilot oxygen mask for Korea Air Force pilots", in Proc. of the 5th International Conference on 3D Body Scanning Technologies, Lugano, Switzerland, 2014, DOI: 10.15221/14.084.

[5] S. M. Weinberg et al., "Digital three-dimensional photogrammetry: Evaluation of anthropometric precision and accuracy using a Genex 3D camera system", in Cleft Palate-Craniofacial Journal, Vol.41, No.5, 2004, pp.507-518, DOI: 10.1597/03-066.1.

[6] R. M. Ball, "3-D design tools from the SizeChina project", in Ergonomics in Design, Vol.17, No.3, 2009, pp.8-13, DOI: 10.1518/106480409X12487281219931.

[7] K. Jung et al., "Evaluation of the multivariate accommodation performance of the grid method", in Applied Ergonomics, Vol.42, 2010, pp.156-161, DOI: 10.1016/j.apergo.2010.06.004.

[8] W. Lee et al., "Development of headforms and an anthropometric sizing analysis system based on 3D head scan images for head-related product designs", in Proc. of Human Factors and Ergonomics Society 59 ${ }^{\text {th }}$ Annual Meeting, Los Angeles, CA, USA, 2015.

[9] Z. Zhuang et al., "Digital 3-D headforms with facial features representative of the current U.S. workforce", in Ergonomics, Vol.53, No.5, 2010, pp.661-671, DOI: 10.1080/00140130903581656.

[10] A. Ballester et L., "3D-based resources fostering the analysis, use, and exploitation of available body anthropometric data", in Proc. of the 5th International Conference on 3D Body Scanning Technologies, Lugano, Switzerland, 2014, DOI: 10.15221/14.237.

[11] J. C. Dai et al., "Sensitivity analysis of important parameters affecting contact pressure between a respirator and a headform", in International Journal of Industrial Ergonomics, Vol.41, No.3, 2011, pp.268-279, DOI: 10.1016/j.ergon.2011.01.007.

[12] A. Jacobson et al., "Bounded biharmonic weights for real-time deformation", in Communications of the Association for Computing Machinery (ACM), Vol.57, No.4, 2014, pp.99-106, DOI: $10.1145 / 2578850$.

[13] Z. Lei et al., "Headform and N95 filtering facepiece respirator interaction: contact pressure simulation and validation", in Journal of Occupational and Environmental Hygiene, Vol.9, 2012, pp.46-58, DOI: 10.1080/15459624.2011.635130.

[14] M. P. Reed et al., "Creating custom avatars for ergonomic analysis using depth cameras", in Proc. of the Human Factors and Ergonomics Society 58th Annual Meeting, Chicago, IL, USA, 2014.

[15] Ergonomic Design Technology Laboratory, http://edt.postech.ac.kr, accessed 2015.

[16] W. Lee et al., "An anthropometric analysis of Korean male helicopter pilots for helicopter cockpit design", in Ergonomics, Vol.56, No.6, 2013, pp.879-887, DOI: 10.1080/00140139.2013.776703.

[17] J. Park et al. "Development of statistical geometric models for a driver's hip and eye locations", in Proc. of the Human Factors and Ergonomics Society 57th Annual Meeting, San Diego, CA, USA, 2013. 
[18] B. Lee et al., "Ergonomic evaluation of a control room design of radioactive waste facility using digital human simulation", in Journal of the Ergonomics Society of Korea, Vol.29, No.3, 2010, pp.383-391, DOI: 10.5143/JESK.2010.29.3.383.

[19] B. Lee et al. "Ergonomic design and evaluation of a console for a counter-battery radar", in Proc. of the 2013 Fall Conference of the Korean Institute of Industrial Engineers, Suwon, Korea, 2013.

[20] E. Jeon et al., "Development of hip protector design process for Korean elderly people", in Proc. of the 2014 Fall Conference of the Korean Institute Society of Fashion \& Textile Industry, Gwangju, Korea, 2014.

[21] H. Jung et al., "Development of an ear measurement method for ear product designs", in Proc. of the 2014 Fall Conference of the Ergonomic Society of Korea, Wonju, Korea, 2014.

[22] K. M. Robinette et al., Civilian American and European Surface Anthropometry Resource (CAESAR) Final Report, Volume I: Summary (AFRL-HE-WP-TR-2002-0169), Wright-Patterson Air Force Base, OH, USA, 2002.

[23] Korean Agency for Technology and Standards (KATS), The Report on the 5th Size-Korea (Korean Body Measurement and Investigation), Ministry of Knowledge Economy, Seoul, Korea, 2004.

[24] ISO, General Requirements for Establishing Anthropometric Databases (ISO 15535), International Standards Organization, Geneva, Switzerland, 2006.

[25] B. Lee et al., "Development of a distributed representative human model (DRHM) generation and analysis system for multiple-size product design", in Proc. of the Human Factors and Ergonomics Society 57th Annual Meeting, San Diego, CA, USA, 2013.

[26] E. Jeon et al., "An ergonomic design of flight suit pattern according to wearing characteristics", in Proc. of the Human Factors and Ergonomics Society 55th Annual Meeting, Las Vegas, CA, USA, 2011.

[27] O. Kwon et al., "Determination of key dimensions for a glove sizing system by analyzing the relationship between hand dimensions", in Applied Ergonomics, Vol.40, No.4, 2009, pp.762-766, DOI: 10.1016/j.apergo.2008.07.003. 